\title{
Dolor abdominal agudo en el adulto mayor: evaluación clínica, diagnóstico y tratamiento
}

\author{
Eduardo Mercado-Cruz ${ }^{1,2,3^{*}}$, M. Alicia Díaz-del Bosque ${ }^{4}$, L. Stephanie Manjarrez-Acevedo ${ }^{5}$ e \\ I. Arely Santiago-Macías ${ }^{6}$
}

${ }^{1}$ Servicio de Urgencias, Unidad de Medicina Familiar N. ${ }^{\circ} 10$, Hospital de Psiquiatría, Instituto Mexicano del Seguro Social; ${ }^{2}$ Facultad de Medicina, Coordinación de Ciclos Clínicos, Universidad Westhill; ${ }^{3}$ Facultad de Medicina, Departamento de Farmacología, Universidad Nacional Autónoma de México; ${ }^{4}$ Unidad de Cuidados Intensivos, Centro Médico Nacional 20 de Noviembre, Instituto de Seguridad y Servicios Sociales de los Trabajadores del Estado; ${ }^{5}$ Servicio de Urgencias, Hospital General de Subzona con Medicina Familiar N. ${ }^{\circ} 41$, Instituto Mexicano del Seguro Social; ${ }^{6}$ Servicio de Urgencias, Hospital General Xoco, Secretaría de Salud de la Ciudad de México. Ciudad de México, México

\section{Resumen}

El dolor abdominal agudo es una causa frecuente por la cual acuden los adultos mayores al servicio de urgencias. En este trabajo se presenta una revisión de la literatura cuyo objetivo fue revisar el cuadro clínico, los auxiliares diagnósticos y el tratamiento en urgencias de los pacientes geriátricos con dolor abdominal agudo. Los cambios fisiológicos asociados al envejecimiento, las comorbilidades y el uso inadecuado de los auxiliares diagnósticos dificulta el abordaje de los adultos mayores con dolor abdominal agudo. La radiografía simple de abdomen es un auxiliar que habitualmente se utiliza en estos pacientes, sin embargo, tiene poca precisión diagnóstica. Los retrasos y errores en el diagnóstico etiológico empeoran el pronóstico y aumentan los costos derivados de la atención médica. El abordaje diagnóstico en urgencias del adulto mayor con dolor abdominal agudo debe centrarse en la reanimación inicial, el control temprano del dolor y la identificación oportuna del diagnóstico etiológico.

Palabras clave: Dolor abdominal agudo. Adulto mayor. Medicina de urgencias.

\section{Acute abdominal pain in elderly patients: clinical evaluation, diagnosis, and treatment}

\section{Abstract}

Acute abdominal pain is a common cause for which older adults present to emergency department. This paper presents a review of literature aimed to review clinical presentation, diagnostic strategies and emergency management of elderly with acute abdominal pain. Physiological changes associated with aging, comorbidities and inappropriate use of diagnostic strategies make it difficult to management older adults with acute abdominal pain. Simple abdominal radiography is a diagnostic procedure commonly used in these patients, however has little diagnostic accuracy. Delays and errors in etiological diagnosis worsen prognosis and increase health care costs. Management in emergency department of elderly with acute abdominal pain should focus on life support, early pain control and timely identification of etiological diagnosis.

Key words: Acute abdominal pain. Elderly. Emergency medicine.

\section{Correspondencia:}

*Eduardo Mercado-Cruz

E-mail: eduardo.mercado.c@zoho.com
Disponible en internet: 16-04-2021

Rev Educ Investig Emer. 2021;3(1):19-28 www.medicinadeemergencias.com

2604-6520 (c) 2021 Sociedad Mexicana de Medicina de Emergencias, AC. Publicado por Permanyer México SA de CV. Este es un artículo open access bajo la licencia CC BY-NC-ND (http://creativecommons.org/licenses/by-nc-nd/4.0/). 


\section{Introducción}

El dolor abdominal es una causa frecuente de consulta en los servicios de urgencias. Se estima que un $60 \%$ de los adultos mayores que acuden a urgencias presentan dolor abdominal como síntoma principal y hasta el $20 \%$ de ellos requerirá tratamiento quirúrgico'.

El deterioro de las reservas metabólicas en los adultos mayores, los cambios fisiológicos (disminución de la agudeza auditiva, percepción alterada del dolor, problemas para la comunicación, respuestas inesperadas en constantes vitales y estudios de laboratorio, entre otros) y las comorbilidades asociadas disminuyen el rendimiento diagnóstico cuando se evalúan adultos mayores con dolor abdominal agudo en el servicio de urgencias $^{1-4}$.

En esta revisión de la literatura presentamos los aspectos más relevantes que tomar en cuenta durante la atención inicial de los adultos mayores que acuden al servicio de urgencias por dolor abdominal agudo.

\section{Método}

Se realizó una revisión de la literatura en Medline empleando términos Mesh. La estrategia de búsqueda utilizada fue: ("Abdominal Pain"[Mesh] OR Non-Traumatic Abdominal Pain AND "Aged"[Mesh] or Elderly AND "Emergency Medicine"[Mesh]). Se obtuvieron un total de 37 referencias bibliográficas. Se incluyeron todas las publicaciones de los últimos 10 años relacionas con la evaluación clínica, estudios de laboratorio e imagen, abordaje diagnóstico y tratamiento en urgencias del adulto mayor con dolor abdominal agudo. Finalmente, 22 de las 37 referencias encontradas cumplieron con los criterios de inclusión de esta revisión bibliográfica.

\section{¿Podemos confiar en las manifestaciones clínicas del adulto mayor con dolor abdominal agudo?}

El dolor abdominal agudo se define como la presencia de dolor abdominal de inicio reciente, constante, intenso y habitualmente acompañado de otros síntomas $^{1,2}$. Después del dolor torácico y la disnea, el dolor abdominal agudo es la causa más frecuente por la cual acuden los adultos mayores al servicio de urgencias y, aunque el dolor abdominal es una manifestación clínica frecuente en el adulto mayor, su atención es más compleja, difícil y costosa en comparación con los adultos jóvenes que acuden por la misma causa ${ }^{2}$.
En orden de frecuencia, las principales causas de dolor abdominal en el adulto mayor son: colecistitis aguda, síndrome de intestino irritable, síndrome ulceroso, oclusión intestinal y diverticulitis ${ }^{2}$. En consecuencia, las intervenciones quirúrgicas de urgencia más realizadas en el adulto mayor son secundarias a enfermedades de la vía biliar, adherencias, hernias, diverticulitis, enfermedades vasculares intestinales, enfermedades malignas y apendicitis ${ }^{1,3,4}$; sin embargo, frecuentemente existen errores en el diagnóstico etiológico del adulto mayor que acude a urgencias por dolor abdominal agudo.

Diversos estudios post mortem han reportado desacuerdos entre el diagnóstico clínico inicial y el anatomopatológico del 28 al 56\% (errores diagnósticos mayores tipo I y || de la clasificación de Goldman, et al.) de los pacientes comparados y algunas de las patologías más subdiagnosticadas (tromboembolia pulmonar, isquemia intestinal, infecciones no reconocidas, infarto agudo de miocardio y disección aórtica) pueden cursar con dolor abdominal agudo como síntoma principal ${ }^{5-9}$.

Los procesos degenerativos de los sistemas neurológico, cardiovascular, renal, gastrointestinal e inmunitario derivados del envejecimiento (Tabla 1) y el uso crónico de medicamentos como antiinflamatorios no esteroideos (AINE), esteroides, bloqueadores de los receptores betaadrenérgicos, bloqueadores de los canales de calcio y antibióticos, entre otros, aumentan la susceptibilidad de los pacientes a patologías gastrointestinales y vasculares, alteran las manifestaciones clínicas, dificultan el interrogatorio y la exploración física, limitan la interpretación de los estudios de laboratorio e incrementan los costos derivados de la atención médica $^{1,2}$.

Las alteraciones cognitivas que suelen presentarse en los adultos mayores dificultan el interrogatorio y la exploración física; por lo tanto, ya sea por medio del interrogatorio directo 0 indirecto (realizado a familiares 0 cuidadores), es necesario recabar información sobre la forma de inicio, localización, características, irradiación, síntomas asociados, enfermedades crónicas y su medicación, y eventos quirúrgicos previos, entre otros. Después del interrogatorio, un examen físico minucioso es fundamental para reducir las posibilidades diagnósticas.

Existen diversas condiciones clínicas extraabdominales que pueden cursar con dolor abdominal (embolia pulmonar, infarto agudo de miocardio, neumonía y cetoacidosis diabética, entre otras), por lo que, además de la exploración abdominal, el examen físico debe incluir otros aspectos como la apariencia, los signos 
Tabla 1. Cambios fisiológicos y patológicos del envejecimiento. Se muestran las principales alteraciones en diferentes aparatos y sistemas asociadas al envejecimiento y su repercusión clínica en los adultos mayores con dolor abdominal agudo

\begin{tabular}{|c|c|c|}
\hline Sistema & Cambios fisiopatológicos & Repercusión clínica \\
\hline Cardiovascular & $\begin{array}{l}\text { Deterioro en las funciones de la pared y el } \\
\text { endotelio vascular } \\
\text { Aumento de la prevalencia de hipertensión arterial, } \\
\text { cardiopatía isquémica e insuficiencia cardiaca } \\
\text { Ingesta crónica de bloqueadores de los receptores } \\
\text { betaadrenérgicos y antagonistas de los canales de } \\
\text { calcio }\end{array}$ & $\begin{array}{l}\text { Incremento de la susceptibilidad a enfermedades } \\
\text { trombóticas y de la pared vascular (embolia pulmonar, } \\
\text { cardiopatía isquémica, patologías aórticas, entre } \\
\text { otras) } \\
\text { Disminución de la respuesta simpática y ausencia de } \\
\text { taquicardia ante diversas condiciones clínicas como } \\
\text { fiebre, dolor e hipovolemia, entre otras }\end{array}$ \\
\hline Inmunológico & $\begin{array}{l}\text { Disminución en la cantidad y calidad de las células } \\
\text { T y B } \\
\text { Disminución de la respuesta a citocinas, antígenos } \\
\text { y pirógenos }\end{array}$ & $\begin{array}{l}\text { Aumenta la sensibilidad a infecciones } \\
\text { Ausencia de leucocitosis y fiebre en presencia en } \\
\text { procesos infecciosos o inflamatorios }\end{array}$ \\
\hline Gastrointestinal & $\begin{array}{l}\text { Disminución del tiempo de llenado gástrico } \\
\text { Aumento de la producción de ácido clorhídrico } \\
\text { Disminución de la función hepática } \\
\text { Formación de divertículos } \\
\text { Disminución de ingesta de agua y nutrientes }\end{array}$ & $\begin{array}{l}\text { Mayor predisposición a la enfermedad ácido-péptica } \\
\text { Disminución del metabolismo de diversos fármacos } \\
\text { Predisposición al estreñimiento e incremento de las } \\
\text { complicaciones derivadas de la enfermedad } \\
\text { diverticular }\end{array}$ \\
\hline Musculo esquelético & $\begin{array}{l}\text { Reducción de la actividad física por disminución de } \\
\text { la masa muscular, densidad ósea y patologías } \\
\text { articulares } \\
\text { Uso crónico de AINE }\end{array}$ & $\begin{array}{l}\text { Incremento del tránsito intestinal y del estreñimiento } \\
\text { Predisposición a la enfermedad ácido-péptica y al } \\
\text { sangrado digestivo alto }\end{array}$ \\
\hline Neurológico & $\begin{array}{l}\text { Deterioro cognitivo } \\
\text { Disminución de la capacidad auditiva } \\
\text { Degeneración del sistema nervioso periférico }\end{array}$ & $\begin{array}{l}\text { Dificultades para realizar el interrogatorio y la } \\
\text { exploración física } \\
\text { Alteraciones en la percepción del dolor y la } \\
\text { temperatura }\end{array}$ \\
\hline $\begin{array}{l}\text { Renal y } \\
\text { genitourinario }\end{array}$ & $\begin{array}{l}\text { Disminución de la filtración glomerular } \\
\text { Menor producción de eritropoyetina } \\
\text { Formación de pequeños divertículos en los túbulos } \\
\text { renales } \\
\text { Menor capacidad para concentrar la orina }\end{array}$ & $\begin{array}{l}\text { Disminución de la eliminación de algunos fármacos y } \\
\text { metabolitos } \\
\text { Predisposición a la anemia } \\
\text { Estasis de orina y crecimiento bacteriano } \\
\text { Predisposición a deshidratación y desequilibrios } \\
\text { hidroelectrolíticos }\end{array}$ \\
\hline
\end{tabular}

AINE: antiinflamatorios no esteroideos.

vitales y la exploración general, con el objetivo de identificar oportunamente tanto las etiologías extraabdominales como las patologías abdominales que habitualmente cursan con inestabilidad cardiovascular (hemorragia gastrointestinal, síndrome aórtico agudo, isquemia mesentérica aguda y perforación intestinal, entre otras) $)^{10-14}$. Las manifestaciones clínicas, los hallazgos en la exploración física y los estudios que solicitar pueden variar significativamente según la etiología ${ }^{15-19}$. A continuación, se muestran las principales patologías que pueden cursar con dolor abdominal agudo en el adulto mayor, así como sus manifestaciones clínicas y la eficacia de los auxiliares diagnósticos (Tabla 2).

Debido a diversos factores, como la pérdida progresiva de las reservas fisiológicas, comorbilidades, procedimientos quirúrgicos previos y retrasos en el reconocimiento de enfermedades graves, la tasa de mortalidad en adultos mayores con dolor abdominal agudo es significativamente más alta en comparación con la de los adultos jóvenes ${ }^{3}$.

La mortalidad de los pacientes geriátricos con dolor abdominal agudo que acuden a urgencias oscila del 11 al $14 \%$, pero esta cifra se incrementa significativamente cuando este grupo de pacientes presenta datos de inestabilidad cardiovascular al momento de su ingre$\mathrm{so}^{2,14,20}$. Por lo tanto, los adultos mayores con dolor abdominal agudo necesitan una atención médica eficiente e inmediata; y, en general, requieren del mismo algoritmo terapéutico que los pacientes inestables por otras causas, es decir, debe priorizarse el control de la vía aérea, la ventilación y la circulación.

De forma paralela, es crucial identificar oportunamente las condiciones clínicas que originan 
Tabla 2. Diagnósticos etiológicos más frecuentes en adultos mayores con dolor abdominal agudo. Se muestran las principales causas abdominales y extraabdominales de dolor abdominal agudo en el adulto mayor

\begin{tabular}{|c|c|c|c|}
\hline Origen & Patología & Características clínicas & Estándar de oro para el diagnóstico \\
\hline \multirow[t]{5}{*}{ Gastrointestinales } & $\begin{array}{l}\text { Colecistitis } \\
\text { aguda }\end{array}$ & $\begin{array}{l}\text { La patología quirúrgica más frecuente en el adulto mayor } \\
\text { Dolor en epigastrio o hipocondrio derecho } \\
\text { Solo el } 53 \% \text { de los pacientes tienen dolor a la } \\
\text { palpación en el cuadrante superior derecho }\end{array}$ & $\begin{array}{l}\text { USG: sensibilidad } 91 \% \text { y } \\
\text { especificidad } 75 \%\end{array}$ \\
\hline & Úlcera péptica & $\begin{array}{l}\text { El } 90 \% \text { de los pacientes tiene el antecedente del } \\
\text { consumo de AINE } \\
\text { El dolor en epigastrio acompañado de dispepsia se } \\
\text { presenta solo en el } 25 \% \text { de los pacientes } \\
\text { La mayoría de los pacientes presenta dolor abdominal } \\
\text { y síntomas inespecíficos } \\
\text { El } 50 \% \text { cursa con perforación }\end{array}$ & $\begin{array}{l}\text { Endoscopia: sensibilidad } 92-98 \% \text { y } \\
\text { especificidad } 30-99 \%\end{array}$ \\
\hline & $\begin{array}{l}\text { Oclusión } \\
\text { intestinal }\end{array}$ & $\begin{array}{l}\text { El } 80 \% \text { cursa con oclusión del intestino delgado } \\
\text { El } 75 \% \text { es secundaria a adherencias por cirugías } \\
\text { abdominales previas } \\
\text { Se caracteriza por distensión, dolor abdominal, } \\
\text { náuseas, vómitos e intolerancia a la vía oral }\end{array}$ & $\begin{array}{l}\text { TC: sensibilidad } 88-99 \% \text { y } \\
\text { especificidad } 96 \%\end{array}$ \\
\hline & Diverticulitis & $\begin{array}{l}\text { La incidencia incrementa con la edad } \\
\text { Típicamente los pacientes acuden por dolor } \\
\text { abdominal en cuadrante interior izquierdo sin vómito }\end{array}$ & $\begin{array}{l}\text { TC: sensibilidad } 93-97 \% \text { y } \\
\text { especificidad } 72-99 \%\end{array}$ \\
\hline & Apendicitis & $\begin{array}{l}\text { Es la tercera patología quirúrgica más frecuente en el } \\
\text { adulto mayor } \\
\text { El } 85 \% \text { de los pacientes se presentan hasta } 24 \text { horas } \\
\text { después del inicio del dolor } \\
\text { El } 25 \% \text { de los pacientes no tiene dolor en el } \\
\text { cuadrante inferior derecho } \\
\text { Solo el } 20 \% \text { presenta la tríada clínica clásica (anorexia, } \\
\text { fiebre y dolor en el cuadrante inferior derecho) }\end{array}$ & $\begin{array}{l}\text { TC con contraste intravenoso: } \\
\text { sensibilidad } 90-99 \% \text { y especificidad } \\
91-99 \% \\
\text { USG sensibilidad } 66-99 \% \text { y } \\
\text { especificidad } 83-96 \%\end{array}$ \\
\hline \multirow[t]{2}{*}{ Vasculares } & $\begin{array}{l}\text { Síndrome } \\
\text { aórtico agudo }\end{array}$ & $\begin{array}{l}\text { El } 10 \% \text { de los hombres mayores de } 65 \text { años tienen } \\
\text { aneurismas de la aorta abdominal no diagnosticados } \\
\text { Es la patología quirúrgica con mayor mortalidad en el } \\
\text { adulto mayor } \\
\text { Solo del } 20 \text { al } 25 \% \text { de los pacientes cursan con la } \\
\text { triada clásica (dolor dorsolumbar y abdominal, } \\
\text { hipotensión y tumoración abdominal pulsátil) } \\
\text { Habitualmente los pacientes cursan con signos de } \\
\text { hipoperfusión tisular e inestabilidad hemodinámica }\end{array}$ & $\begin{array}{l}\text { Angiotomografía: sensibilidad } 95 \% \text { y } \\
\text { especificidad } 87-99 \% \\
\text { USG: sensibilidad } 87 \% \text { y } \\
\text { especificidad } 99 \%\end{array}$ \\
\hline & $\begin{array}{l}\text { Isquemia } \\
\text { mesentérica } \\
\text { aguda }\end{array}$ & $\begin{array}{l}\text { Es la segunda patología quirúrgica con mayor } \\
\text { mortalidad en el adulto mayor } \\
\text { Habitualmente cursa con sintomatología inespecífica, } \\
\text { lo cual dificulta su diagnóstico } \\
\text { Los pacientes pueden cursar con dolor abdominal } \\
\text { generalizado, vómitos y diarrea }\end{array}$ & $\begin{array}{l}\text { Angiotomografía: sensibilidad } 93 \% \text { y } \\
\text { especificidad } 95 \%\end{array}$ \\
\hline Inflamatorias & $\begin{array}{l}\text { Pancreatitis } \\
\text { aguda }\end{array}$ & $\begin{array}{l}\text { En el } 75 \% \text { de los casos es secundaria a litiasis } \\
\text { vesicular o de la vía biliar } \\
\text { El } 90 \% \text { de los adultos mayores no presenta dolor } \\
\text { abdominal típico } \\
\text { Habitualmente los pacientes presentan fiebre, } \\
\text { náuseas, vómitos e ictericia } \\
\text { El dolor abdominal típico está ausente en el } 90 \% \text { de los } \\
\text { casos de pancreatitis en adultos mayores de } 65 \text { años }\end{array}$ & $\begin{array}{l}\text { TC: sensibilidad } 78 \% \text { y especificidad } \\
86 \%\end{array}$ \\
\hline \multirow[t]{4}{*}{ Extraabdominales } & Torácicas & $\begin{array}{l}\text { Síndromes coronarios agudos, tromboembolia } \\
\text { pulmonar, neumonía basal, neumotórax y pericarditis }\end{array}$ & Depende de cada patología \\
\hline & Metabólicas & $\begin{array}{l}\text { Uremia, insuficiencia suprarrenal aguda y } \\
\text { cetoacidosis diabética }\end{array}$ & Depende de cada patología \\
\hline & Hematológicas & Leucemia aguda y crisis hemolíticas & Depende de cada patología \\
\hline & Neurológicas & Herpes zóster y dolor radicular & Depende de cada patología \\
\hline
\end{tabular}


hipotensión arterial e hipoperfusión tisular con el objetivo de reconocer a los pacientes que serán beneficiados de una terapia quirúrgica inmediata (síndrome aórtico agudo, trombosis mesentérica, perforación intestinal).

El objetivo primordial en los adultos mayores con dolor abdominal que cursan con inestabilidad es brindar una adecuada reanimación inicial sin retrasar el tratamiento definitivo; en general, el interrogatorio, la exploración física, un alto índice de sospecha y la ultrasonografía en la cabecera del paciente son suficientes para determinar el tratamiento que requerirá el paciente. Aunque la tomografía tiene mayor precisión diagnóstica que el ultrasonido, debe evitarse en pacientes inestables, porque además de requerir que el paciente sea trasladado a la sala de radiodiagnóstico, suele asociarse con desenlaces fatales porque genera retrasos en el diagnóstico y tratamiento definitivo.

\section{¿Debemos solicitar radiografías simples de abdomen como primer estudio de imagen en el adulto mayor con dolor abdominal agudo que acude a urgencias?}

Los estudios de imagen juegan un papel crucial en la evaluación de pacientes con dolor abdominal agudo. La selección del estudio adecuado ayuda a determinar el diagnóstico etiológico, identificar quiénes requerirán de alguna intervención quirúrgica urgente y seleccionar aquellos pacientes que pueden ser manejados de forma segura sin necesidad de ser hospitalizados.

Tradicionalmente, la evaluación inicial del adulto mayor con dolor abdominal agudo incluye la realización sistemática de radiografías simples de abdomen en dos o más posiciones; sin embargo, cada vez hay mayor evidencia sobre la poca utilidad de esta herramienta diagnóstica ${ }^{21-23}$.

Hace algunas décadas, la radiografía de abdomen era el único estudio de imagen disponible para la evaluación de los pacientes con dolor abdominal. En la actualidad, diversas publicaciones han demostrado la baja sensibilidad y especificidad de este método diagnóstico ${ }^{23-25}$, incluso en situaciones donde se creía que era muy útil, como el neumoperitoneo, litiasis del tracto urinario, detección de cuerpos extraños y oclusión intestinal ${ }^{23,26}$. Además, hasta el $70 \%$ de los pacientes con dolor abdominal agudo pueden tener una radiografía de abdomen normal ${ }^{23}$.

Por lo tanto, las radiografías simples de abdomen no contribuyen a la certeza diagnóstica, retrasan la toma de decisiones y el tratamiento definitivo, someten a los pacientes a una radiación innecesaria e incrementan los costos derivados de la atención médica.

En las últimas dos décadas, la disponibilidad y el uso de otros métodos de imagen como el ultrasonido y la tomografía han incrementado significativamente, en consecuencia, ha mejorado la precisión diagnóstica durante la evaluación inicial de los adultos mayores con dolor abdominal agudo que acuden a urgencias ${ }^{27}$. En este sentido, la ultrasonografía en la cabecera del paciente es una herramienta diagnóstica extremadamente útil porque permite identificar la presencia de líquido libre en la cavidad peritoneal, estimar el volumen intravascular, valorar la aorta abdominal y reconocer patologías extraabdominales causantes de inestabilidad (síndromes coronarios agudos, derrame pleural masivo, embolia pulmonar, taponamiento cardiaco y neumotórax, entre otras) mediante la valoración del parénquima pulmonar, espacios pleurales, vasos sanguíneos y corazón ${ }^{28,29}$.

Diversos estudios han evidenciado que el ultrasonido y la tomografía computarizada después de una ultrasonografía abdominal no concluyente son significativamente superiores en términos de precisión diagnóstica, cuando se comparan con la radiografía simple de abdomen ${ }^{17,23,25}$. Además, el uso temprano de la tomografía como método diagnóstico en adultos mayores con dolor abdominal agudo ha demostrado mejorar la toma de decisiones, ayudar a la planeación quirúrgica en caso de ser necesaria y disminuir tanto el tiempo de estancia de los pacientes en las salas de urgencias, como el número de cirugías innecesarias ${ }^{27}$.

Aunque la tomografía computarizada ha demostrado mejores resultados cuando se compara con otras estrategias diagnósticas, la evidencia disponible en la literatura recomienda usar el ultrasonido como primer estudio de imagen durante la evaluación en urgencias de pacientes con dolor abdominal agudo y reservar la tomografía ante una ultrasonografía abdominal no concluyente. Esto debido a que diversos estudios documentaron que el uso de la tomografía computarizada simple después de una ultrasonografía abdominal negativa disminuye hasta el $50 \%$ de las tomografías realizadas, reduce la exposición a radiación y tiene una precisión diagnóstica similar al uso inicial de la tomografía $17,23,25$.

A pesar de la disponibilidad de diversas herramientas, la evaluación inicial del adulto mayor con dolor abdominal agudo y la determinación del diagnóstico etiológico continúan siendo un reto aún para un médico experimentado; por lo tanto, dada la baja sensibilidad y especificad de la radiografía de abdomen, la sintomatología atípica que pueden manifestar los adultos 
Tabla 3. Estudios de imagen para el abordaje diagnóstico del adulto mayor con dolor abdominal agudo. Se muestran las ventajas, desventajas e indicaciones de las herramientas de imagen que pueden ser utilizadas durante el abordaje de adultos mayores con dolor abdominal agudo

\begin{tabular}{|c|c|c|c|}
\hline Estudio & Usos apropiados & Ventajas & Desventajas \\
\hline $\begin{array}{l}\text { Radiografías } \\
\text { simples de } \\
\text { abdomen }\end{array}$ & $\begin{array}{l}\text { Identificación y seguimiento de } \\
\text { cuerpos extraños radiopacos } \\
\text { Seguimiento de pacientes con } \\
\text { oclusión intestinal } \\
\text { Localización de catéteres }\end{array}$ & Bajo costo & $\begin{array}{l}\text { Poca precisión diagnóstica } \\
\text { Frecuentemente son normales o } \\
\text { tienen hallazgos inespecíficos }\end{array}$ \\
\hline $\begin{array}{l}\text { Ultrasonido } \\
\text { abdominal }\end{array}$ & $\begin{array}{l}\text { Diagnóstico de patologías vesiculares } \\
\text { y de la vía biliar, hepáticas, } \\
\text { esplénicas, ginecológicas y aórticas }\end{array}$ & $\begin{array}{l}\text { Bajo costo } \\
\text { No invasivo } \\
\text { Puede realizarse en la } \\
\text { cabecera del paciente }\end{array}$ & $\begin{array}{l}\text { Dependiente del operador } \\
\text { Poca precisión diagnóstica en } \\
\text { pancreatitis, patologías intestinales y } \\
\text { neumoperitoneo }\end{array}$ \\
\hline $\begin{array}{l}\text { Tomografía } \\
\text { computarizada }\end{array}$ & $\begin{array}{l}\text { Diagnóstico de pancreatitis, } \\
\text { obstrucción intestinal, apendicitis, } \\
\text { diverticulitis, neumoperitoneo, litiasis } \\
\text { renal y en el tracto urinario, tumores, } \\
\text { abscesos y patologías } \\
\text { vasculares (síndromes aórticos agudos } \\
\text { e isquemia mesentérica aguda) }\end{array}$ & $\begin{array}{l}\text { Alta precisión diagnóstica } \\
\text { Mejora la toma de decisiones } \\
\text { Ayuda la planeación quirúrgica } \\
\text { en caso de ser necesaria } \\
\text { Útil como guía para el drenaje } \\
\text { de colecciones }\end{array}$ & $\begin{array}{l}\text { Requiere movilizar al paciente a la } \\
\text { sala de radiodiagnóstico } \\
\text { En ocasiones es necesario administrar } \\
\text { contraste intravenoso, oral o ambos } \\
\text { Riesgo de reacciones adversas } \\
\text { asociadas al medio de } \\
\text { contraste (anafilaxia y nefrotoxicidad) }\end{array}$ \\
\hline
\end{tabular}

mayores y las consecuencias negativas de los retrasos en el diagnóstico etiológico, es recomendable realizar los esfuerzos necesarios para abandonar la práctica rutinaria de solicitar radiografías de abdomen durante la evaluación del adulto mayor con dolor abdominal agudo que acude a un servicio de urgencias.

El estudio de imagen para la evaluación del adulto mayor con dolor abdominal agudo debe elegirse con base en diversos factores como el estado clínico del paciente, diagnóstico etiológico presuntivo y la precisión, riesgos y beneficios del estudio que solicitar (Tabla 3); con los objetivos de determinar un diagnóstico etiológico, ofrecer un tratamiento oportuno y disminuir tanto los procedimientos innecesarios, como los costos derivados de la estancia prolongada de los pacientes en un servicio de urgencias.

\section{¿Cuándo podemos iniciar la analgesia en adultos mayores con dolor abdominal agudo?}

Tradicionalmente la administración de analgésicos en pacientes con dolor abdominal agudo es retrasada hasta establecer el diagnóstico etiológico definitivo. Hasta el $20 \%$ de los adultos mayores con dolor abdominal agudo no reciben analgésicos durante su estancia en los servicios de urgencias ${ }^{1,2}$.

El dolor abdominal agudo en el adulto mayor tiene diversos efectos negativos sobre diversos aparatos y sistemas; la mayoría de ellos están vinculados fundamentalmente con la liberación de catecolaminas. En el sistema cardiovascular, el dolor produce taquicardia y aumento de las resistencias vasculares periféricas, que a su vez generan hipertensión arterial, vasoconstricción coronaria e inducen un aumento en la demanda y el consumo de $\mathrm{O}_{2}$ por el tejido miocárdico. Estos elementos precipitan la isquemia miocárdica y el desarrollo de arritmias ${ }^{30,31}$.

En el sistema respiratorio, el dolor abdominal agudo produce contracturas musculares y disfunción diafragmática, disminuye la expansión torácica y el reflejo tusígeno, altera la ventilación y conduce a hipoxemia, atelectasias y neumonía. En los órganos intraabdominales disminuye la movilidad del músculo liso, aumenta el tono de esfínteres y genera distensión gástrica, acumulación de secreciones y vasoconstricción esplácnica; dichas alteraciones se manifiestan con náuseas, vómitos, íleo y retención aguda de orina. Además, también produce neutropenia y disfunción de la respuesta inmunitaria celular y humoral ${ }^{30-32}$.

Pese a que los efectos negativos del dolor abdominal agudo sobre los sistemas cardiovascular, respiratorio, gastrointestinal e inmunitario son ampliamente conocidos, la analgesia en urgencias es habitualmente retrasada hasta la valoración por cirugía general o el establecimiento del diagnóstico etiológico definitivo por la falsa creencia de que los analgésicos disminuyen la precisión diagnóstica.

La evidencia disponible en la literatura apoya la administración temprana de analgésicos en pacientes con dolor abdominal agudo. Diversos estudios han documentado que la analgesia no disminuye la precisión 
diagnóstica y, por el contrario, facilita el interrogatorio, permite al clínico realizar una mejor exploración física y aumenta el grado satisfacción del paciente ${ }^{33,34}$. Por lo tanto, independientemente de si se conoce o no el diagnóstico etiológico, es recomendable administrar analgésicos desde la valoración inicial del adulto mayor con dolor abdominal agudo que acude a urgencias.

\section{¿Cuál es el mejor analgésico para el adulto mayor con dolor abdominal agudo?}

La analgesia es una pieza crucial en el tratamiento del adulto mayor con dolor abdominal agudo que acude a urgencias y debe ser administrada lo más pronto posible. Aunque una analgesia inadecuada está asociada con resultados negativos como mayor tiempo de estancia hospitalaria, retraso en la deambulación, delirio y dolor crónico ${ }^{35}$; en lo general, los adultos mayores reciben menos analgésicos que los adultos jóvenes $^{36}$. Los objetivos de la administración temprana de analgésicos en los adultos mayores con dolor abdominal agudo son aliviar la sintomatología, disminuir los efectos sistémicos del dolor agudo y evitar las reacciones adversas de los analgésicos.

La valoración cuidadosa con reevaluación frecuente permite al médico brindar una analgesia eficaz y segura. La escala verbal numérica es la herramienta más utilizada para evaluar la intensidad del dolor porque es efectiva y fácil de aplicar; sin embargo, su uso está restringido a pacientes sin alteraciones neurológicas ${ }^{35}$. Aunque la evaluación del dolor en adultos mayores puede estar limitada por las alteraciones cognitivas que frecuentemente se presentan en este grupo etario, existen algunas escalas diseñadas para valorar el dolor en pacientes con alteraciones neurológicas que combinan la información procedente del interrogatorio con las expresiones faciales y los comportamientos del paciente. Algunas de estas escalas son: Abbey Pain Scale, Checklist of nonverbal pain indicators, Pain Assessment Checklist for Seniors with Limited Ability to Communicate (PACSLAC), Critical-care pain observation tool (CPOT) y Algoplus $2,35,37$.

Actualmente no existen pautas con respecto al tratamiento analgésico en adultos mayores con dolor abdominal agudo, en gran medida porque la mayoría de los estudios clínicos aleatorizados excluyen tanto a los adultos mayores como a los pacientes con múltiples comorbilidades.

Los adultos mayores tienen un riesgo del 10 al 25\% mayor que los adultos jóvenes de presentar efectos adversos asociados a medicamentos ${ }^{38}$. Dado que la disminución en el funcionamiento renal y hepático, los cambios en la composición corporal, las enfermedades crónicas y la polifarmacia aumentan el riesgo de desarrollar efectos farmacológicos adversos ${ }^{30,39}$, el uso de analgésicos en los adultos mayores deberá iniciarse con las dosis mínimas necesarias para obtener una analgesia eficaz sin incrementar el riesgo de efectos secundarios indeseados (sedación excesiva, depresión respiratoria, lesión renal aguda y hemorragia gastrointestinal, entre otros) y, en caso de ser necesario, los incrementos en la dosificación deberán realizarse lentamente.

El paracetamol y los opioides son útiles para el control del dolor abdominal en niños y adultos jóvenes porque además de ser altamente eficaces, han demostrado que no disminuyen la precisión diagnósti$\mathrm{ca}^{33,34,40,41}$. En general, los AGS Beers Criteria (Criterios Beers de la Sociedad Americana de Geriatría) consideran que la mayoría de los AINE son medicamentos inapropiados en los adultos mayores porque frecuentemente se han asociado a eventos cardiovasculares adversos, hemorragia gastrointestinal y lesión renal aguda $^{42}$.

El paracetamol es el medicamento más seguro para el manejo del adulto mayor con dolor agudo porque no está asociado a los efectos secundarios de los AINE y, según la Asociación Americana de Geriatría, además de ser la primera línea para el tratamiento de pacientes con dolor leve a moderado, es un componente esencial de la analgesia multimodal ${ }^{35,42}$.

Para adultos mayores con dolor agudo moderado a severo, los opioides son la mejor opción; sin embargo, su administración debe ser cautelosa porque habitualmente requieren menores dosis que los adultos jóvenes para obtener un adecuado control del dolor y el riesgo de efectos adversos es significativamente más alto ${ }^{35,37}$.

\section{¿Qué decisiones se deben tomar el adulto mayor con dolor abdominal agudo?}

En la figura 1 se propone un algoritmo diagnóstico y terapéutico que puede ser utilizado en el adulto mayor con dolor abdominal agudo y en la tabla 4 se mencionan algunos de los medicamentos con sus respectivas dosis que pueden ser administrados para el control del dolor de estos enfermos. Finalmente, en la tabla 5 se muestran los puntos más importantes que deben ser tomados en cuenta durante la atención en el servicio 


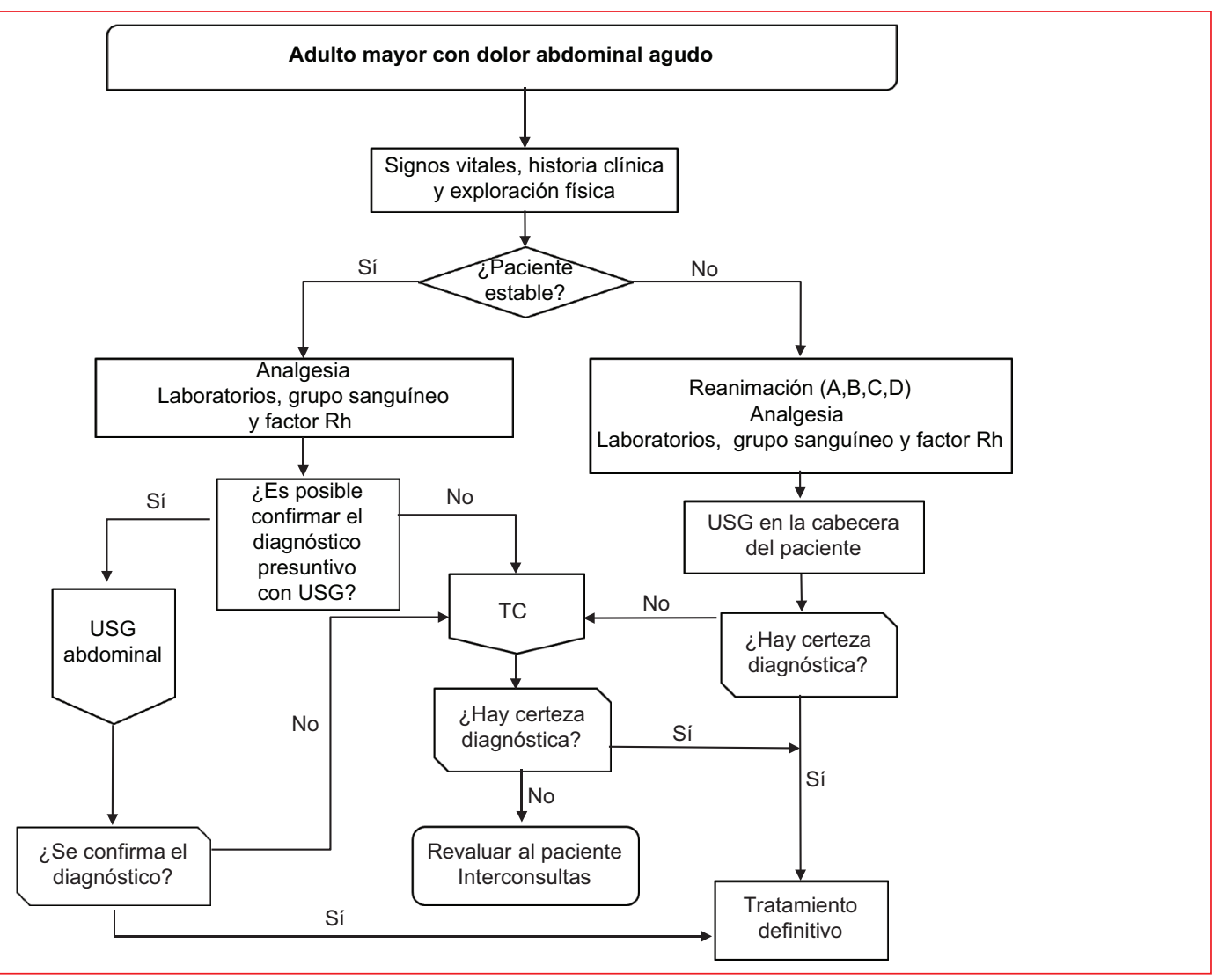

Figura 1. Algoritmo para el diagnóstico y tratamiento de adultos mayores con dolor abdominal agudo. USG: ultrasonografía; TC: tomografía computarizada.

Tabla 4. Analgésicos para el adulto mayor con dolor abdominal agudo. Se muestran las dosis de los principales analgésicos que pueden ser utilizados para el control del dolor abdominal agudo en adultos mayores

\begin{tabular}{|c|c|c|}
\hline Analgésico & Dosis recomendada & Consideraciones \\
\hline Paracetamol & $\begin{array}{l}325-500 \mathrm{mg} \text { V0 cada } 4 \text { horas o } \\
500-1,000 \mathrm{mg} \text { IV cada } 6 \text { u } 8 \text { horas }\end{array}$ & $\begin{array}{l}\text { Analgésico de primera elección en el adulto mayor } \\
\text { Además de tener potentes efectos analgésicos y antipiréticos, } \\
\text { es el medicamento con el perfil farmacológico más seguro }\end{array}$ \\
\hline Ibuprofeno & $200 \mathrm{mg}$ V0 cada 8 horas & $\begin{array}{l}\text { Prescribir solo si el beneficio supera los riesgos (los AGS } \\
\text { Beers Criteria recomiendan evitar su uso). No administrar por } \\
\text { tiempos prolongados } \\
\text { Es posible agregar un inhibidor de la bomba de protones para } \\
\text { disminuir sus efectos adversos gastrointestinales }\end{array}$ \\
\hline Naproxeno & $250 \mathrm{mg}$ V0 cada 8 o 12 horas & $\begin{array}{l}\text { Prescribir solo si el beneficio supera los riesgos (los AGS } \\
\text { Beers Criteria recomiendan evitar su uso). No administrar por } \\
\text { tiempos prolongados } \\
\text { Es posible agregar un inhibidor de bomba de protones para } \\
\text { disminuir sus efectos adversos gastrointestinales }\end{array}$ \\
\hline Tramadol & $\begin{array}{l}50-100 \mathrm{mg} \text { V0 o IV cada } 6 \text { u } 8 \text { horas } \\
\text { Dosis máxima } 400 \mathrm{mg} \text { en } 24 \text { horas ( } 300 \mathrm{mg} \text { en } \\
\text { mayores de } 75 \text { años y } 200 \mathrm{mg} \text { en pacientes con } \\
\text { FG menor de } 30 \mathrm{ml} / \mathrm{min} \text { ) }\end{array}$ & $\begin{array}{l}\text { Puede exacerbar o causar SIADH e hiponatremia } \\
\text { Vigilar el nivel de sodio al inicio y durante el tratamiento }\end{array}$ \\
\hline Morfina & $1.5-2.5 \mathrm{mg}$ IV cada 4 horas & $\begin{array}{l}\text { No hay dosis máxima (mientras se obtenga un efecto } \\
\text { analgésico sin efectos secundarios) } \\
\text { Los incrementos deben ser lentos (aproximadamente del } 20 \text { al } \\
25 \% \text { de la dosis inicial) } \\
\text { Si la condición clínica del paciente lo permite, es posible } \\
\text { añadir laxantes, antieméticos y una adecuada hidratación para } \\
\text { disminuir sus efectos secundarios }\end{array}$ \\
\hline
\end{tabular}

V0: vía oral; IV: intravenoso; AGS Beers Criteria: criterios Beers de la Sociedad Americana de Geriatría; FG: filtración glomerular; SIADH: síndrome de secreción inadecuada de la hormona antidiurética. 
Tabla 5. Puntos clave para el abordaje de adultos mayores con dolor abdominal agudo

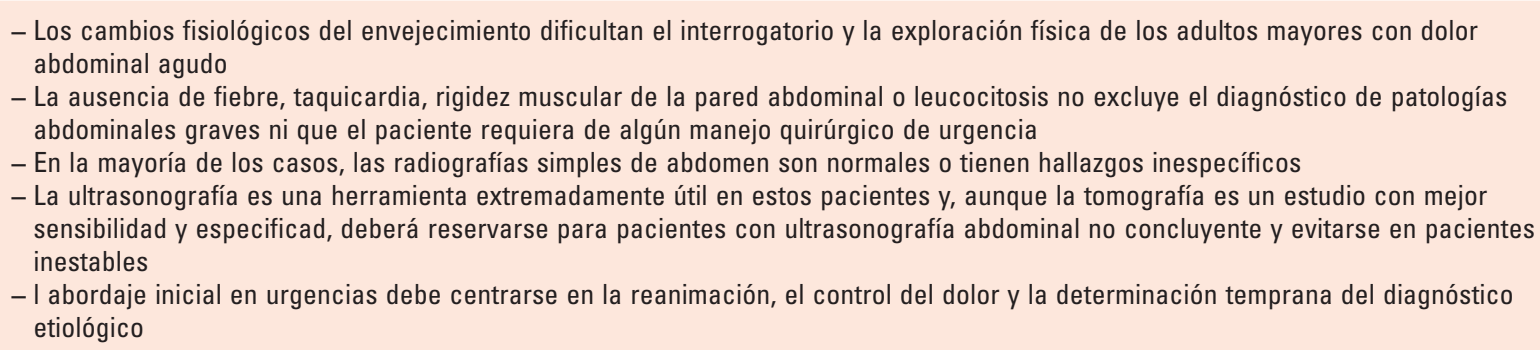

de urgencias de los pacientes geriátricos con dolor abdominal agudo.

\section{Conclusiones}

La pérdida de las reservas fisiológicas, las comorbilidades y la polifarmacia son factores que dificultan el abordaje diagnóstico del adulto mayor que acude a urgencias por dolor abdominal agudo. La radiografía simple de abdomen es una herramienta poco útil en estos pacientes. La ultrasonografía abdominal y la tomografía facilitan la identificación oportuna del diagnóstico etiológico. Los adultos mayores con dolor abdominal agudo tienen alta tasa de mortalidad y su tratamiento en urgencias debe centrarse en la reanimación inicial, el control del dolor y la identificación temprana del diagnóstico etiológico, con el objetivo de disminuir retrasos en el tratamiento definitivo.

\section{Financiamiento}

La presente investigación no ha recibido ningunabeca específica de agencias de los sectores públicos, comercial, o sin ánimo de lucro.

\section{Conflicto de intereses}

Los autores declaran no tener conflicto de intereses alguno.

\section{Responsabilidades éticas}

Protección de personas y animales. Los autores declaran que para esta investigación no se han realizado experimentos en seres humanos ni en animales.

Confidencialidad de los datos. Los autores declaran que en este artículo no aparecen datos de pacientes.
Derecho a la privacidad y consentimiento informado. Los autores declaran que en este artículo no aparecen datos de pacientes.

\section{Bibliografía}

1. Magidson PD, Martinez JP. Abdominal pain in the geriatric patient. Emerg Med Clin North Am. 2016;34(3):559-74.

2. Ragsdale $L$, Southerland $L$. Acute abdominal pain in the older adult. Emerg Med Clin North Am. 2011;29(2):429-48.

3. Henden Çam P, Baydin A, Yürüker S, Erenler AK, 区engüldür E. Investigation of geriatric patients with abdominal pain admitted to Emergency Department. Curr Gerontol Geriatr Res. 2018:2018(1):1-8.

4. Pérez-Hernández JL, Teuffer-Carrion LT, Díaz-Aldana EV, HernándezGuerrero S, Moron-Ventura P, Nieves-Duran M, et al. Dolor abdominal agudo en pacientes geriátricos evaluados en el servicio de urgencias en un hospital de tercer nivel. Rev Gastroenterol Mex. 2010;75(3):261-6.

5. Fares AF, Fares J, Fares GF, Cordeiro JA, Nakazone MA, Cury PM. Clinical and pathological discrepancies and cardiovascular findings in 409 consecutive autopsies. Arq Bras Cardiol. 2011;97(6):449-55.

6. Ermenc B. Comparison of the clinical and post mortem diagnoses of the causes of death. Forensic Sci Int. 2000;114(2):117-9.

7. Aalten CM, Samson MM, Jansen PAF. Diagnostic errors; the need to have autopsies. Neth J Med. 2006;64(6):186-90.

8. Bürgesser MV, Camps D, Calafat P, Diller ANA. Discrepancias entre diagnósticos clínicos y hallazgos de autopsia. Med (Buenos Aires). 2011;2(71):135-8.

9. Magret Iglesias M, Vidaur Tello L, Fernández Olsina S, García Fontgivell JF, Blázquez Vilàs $S$, Alonso Rubio $S$, et al. Discrepancias entre el diagnóstico clínico y el anatomopatológico en un servicio de cuidados intensivos polivalente. Med Intensiva. 2006;30(3):95-100.

10. Enríquez Vega M, Solorio Rosete H, Cossío Zazueta A, Bizueto Rosas H, Cruz Castillo J, Iturburu Enríquez A. Detección oportuna de aneurismas de aorta abdominal en población de riesgo. Rev Med Inst Mex Seguro Soc. 2015;53:100-3.

11. Hawthorn BR, Ratnam LA. Acute mesenteric ischaemia: imaging and intervention. Clin Radiol. 2019;75(5):1-10.

12. Barmase M, Kang M, Wig J, Kochhar R, Gupta R, Khandelwal N. Role of multidetector CT angiography in the evaluation of suspected mesenteric ischemia. Eur J Radiol. 2011;80(3):e582-7.

13. Mussa FF, Horton JD, Moridzadeh R, Nicholson J, Trimarchi S, Eagle KA. Acute aortic dissection and intramural hematoma. JAMA. 2016;316(7):754.

14. Spangler R, van Pham T, Khoujah D, Martinez JP. Abdominal emergencies in the geriatric patient. Int J Emerg Med. 2014;7(43):1-8.

15. Hernández Paz DJR, Guerrero Avendaño G. Tomografía computada multicorte y su utilidad en el diagnóstico de obstrucción intestinal en pacientes del Hospital General de México. An Radiol México. 2012; 4:246-53.

16. Cano Muñoz I, Flores Salinas MA. Utilidad de la tomografía computada en el diagnóstico de diverticulitis, su estadiaje y tratamiento médico-quirúrgico según la escala de Minnesota. An Radiol México. 2010; 9(3):130-6.

17. Arévalo Espejo O de J, Moreno Mejía ME, Ulloa Guerrero LH. Apendicitis aguda: Hallazgos radiológicos y enfoque actual de las imágenes diagnósticas. Rev Colomb Radiol. 2014;25(1):3877-88.

18. Knight AL, Howlett DC. Imaging of the acute abdomen. Surgery. 2010;28(11):568-73. 
19. Payor A, Jois P, Wilson J, Kedar R, Nallamshetty L, Grubb S, et al. Efficacy of noncontrast computed tomography of the abdomen and pelvis for evaluating nontraumatic acute abdominal pain in the emergency department. J Emerg Med. 2015;49(6):886-92.

20. McNamara R, Dean AJ. Approach to acute abdominal pain. Emerg Med Clin North Am. 2011;29(2):159-73.

21. Van Randen A, Laméris W, Luitse JSK, Gorzeman M, Hesselink EJ, Dolmans DEJGJ, et al. The role of plain radiographs in patients with acute abdominal pain at the ED. Am J Emerg Med. 2011;29(6):582-589.e2.

22. Artigas Martín JM, Martí de Gracia M, Rodríguez Torres C, Marquina Martínez D, Parrilla Herranz P. Routine abdominal X-rays in the emergency department: A thing of the past? Radiol. 2015;57(5):380-90.

23. Gans SL, Stoker J, Boermeester MA. Plain abdominal radiography in acute abdominal pain; past, present, and future. Int J Gen Med. 2012;5:525-33.

24. Patel NK, Hatrick A. Imaging and radiological interventions for the acute abdomen. Surgery [Internet]. 2013;31(11):588-96. Disponible en: https:// doi.org/10.1016/..mpsur.2013.08.010

25. Laméris W, van Randen A, Wouter van Es H, van Heesewijk JPM, van Ramshorst B, Bouma WH, et al. Imaging strategies for detection of urgen conditions in patients with acute abdominal pain: Diagnostic accuracy study. BMJ. 2009;339(7711):29-33.

26. Lee JH, Kim HC, Yang DM, Kim SW, Jin W, Park SJ, et al. What is the role of plain radiography in patients with foreign bodies in the gastrointestinal tract? Clin Imaging. 2012;36(5):447-54.

27. Gardner CS, Jaffe TA, Nelson RC. Impact of CT in elderly patients presenting to the emergency department with acute abdominal pain. Abdom Imaging. 2015;40(7):2877-82.

28. Whitson MR, Mayo PH. Ultrasonography in the emergency department Crit Care. 2016:20(1):1-8.

29. Blanco P. Aguiar FM, Blaivas M. Rapid ultrasound in shock (RUSH) velocity-time integral: A proposal to expand the RUSH protocol. $J$ Ultrasound Med. 2015;34(9):1691-700.

30. Ellison DL. Physiology of pain. Crit Care Nurs Clin North Am. 2017; 29(4):397-406.
31. Perez T, Castañeda J. Fisiopatología del dolor agudo: alteraciones cardiovasculares, respiratorias y de otros sistemas y órganos. Rev Cuba Anestesiol Reanim. 2012;11(1):19-26.

32. Hudspith MJ. Anatomy, physiology and pharmacology of pain. Anaesth Intensive Care Med. 2016;17(9):425-30.

33. Manterola C, Vial M, Moraga J, Astudillo P. Analgesia in patients with acute abdominal pain. Cochrane Database Syst Rev. 2011:(1):CD005660.

34. Falch C, Vicente D, Häberle H, Kirschniak A, Müller S, Nissan A, et al. Treatment of acute abdominal pain in the emergency room: A systematic review of the literature. Eur J Pain. 2014;18(7):902-13.

35. Hwang U, Platts-Mills TF. Acute pain management in older adults in the Emergency Department. Clin Geriatr Med. 2013;29(1):151-64.

36. Platts-Mills TF, Esserman DA, Brown DL, Bortsov AV, Sloane PD, McLean SA. Older US Emergency Department patients are less likely to receive pain medication than younger patients: Results from a national survey. Ann Emerg Med. 2012;60(2):199-206.

37. Malcolm C. Acute pain management in the older person. J Perioper Pract. 2015;25(7-8):134-9.

38. Sáez López MP, Sánchez Hernández N, Jiménez Mola S, Alonso García N, Valverde García JA. Tratamiento del dolor en el anciano: Opioides y adyuvantes. Rev Soc Esp Dolor. 2016;23(2):93-104.

39. Farrar FC, White D, Darnell L. Pharmacologic interventions for pain management. Crit Care Nurs Clin North Am. 2017;29(4):427-47.

40. Gavriilidis P, de'Angelis N, Tobias A. To use or not to use opioid analgesia for acute abdominal pain before definitive surgical diagnosis? A Systematic Review and Network Meta-Analysis. J Clin Med Res. 2019;11(2):121-6.

41. Oguzturk H, Ozgur D, Turtay MG, Kayaalp C, Yilmaz S, Dogan M, et al. Tramadol or paracetamol do not effect the diagnostic accuracy of acute abdominal pain with significant pain relief. Eur Rev Med Pharmacol Sci. 2012;16:1983-8.

42. Fick DM, Semla TP, Steinman M, Beizer J, Brandt N, Dombrowski R, et al. American Geriatrics Society 2019 Updated AGS Beers Criteria® for Potentially Inappropriate Medication Use in Older Adults. J Am Geriatr Soc. 2019;67(4):674-94. 